\title{
V652 Her: Evidence that White Dwarf Mergers Really do Happen
}

\author{
C. Simon Jeffery \\ Armagh Observatory, College Hill, Armagh BT61 9DG, Northern \\ Ireland
}

\begin{abstract}
V652 Herculis is a pulsating B star with a mass of about $0.6 \mathrm{M}_{\odot}$ and a luminosity of some $1000 \mathrm{~L}_{\odot}$. Its uniqueness is defined by a surface which is predominantly $(99 \%)$ CNO-processed helium. Precise measurements of its pulsation properties have provided direct tests for stellar pulsation and evolution models. Simulations of the merger of two helium white dwarfs are very successful in explaining its origin.
\end{abstract}

\section{Introduction}

$\mathrm{V} 652 \mathrm{Her}=\mathrm{BD}+13^{\circ} 3224$ is unusual, even for an extreme helium star. The latter are rare low-mass $A$ and $B$ supergiants. The majority have the dimensions of post-asymptotic-giant-branch stars and show no evidence for planetary nebulae. None have binary companions. Hydrogen lines are either weak or absent and most are carbon rich. V652 Her is less luminous than the majority, carbon poor and visibly shrinking. Its properties have been explored in considerable detail and point to an exotic past. This review is based primarily on work published by Saio \& Jeffery (2000), Jeffery, Woolf, \& Pollacco (2001), and Montañéz Rodríguez \& Jeffery (2002).

\section{V652 Her}

V652 Her was found to be a helium star, comparable to HD 124448, HD 168476 and $\mathrm{BD}+10^{\circ} 2179$, by Berger \& Greenstein (1953). Landolt (1973) discovered light variations with a period of $0.108 \mathrm{~d}$ that were similar to those then being found in $\beta$ Cepheid stars, but it was obviously not a population I main-sequence star. Radial-velocity studies (Hill et al. 1981) showed V652 Her to be a pulsating variable, from which Lynas-Gray et al. (1984) were able to measure a radius using Baade's method. With the spectroscopically measured surface gravity, they deduced a mass of $0.7_{-0.3}^{+0.4} \mathrm{M}_{\odot}$ and a luminosity about $1000 \mathrm{~L}_{\odot}$.

Whilst refining the light curve and ephemeris for V652 Her, Kilkenny et al. (1982) discovered that the pulsation period was decreasing at a substantial rate. Subsequent observations have established that a quartic ephemeris is necessary to model the secular change in period (Kilkenny, Lynas-Gray, \& Roberts 1996). The linear term corresponds to secular contraction of the star on a timescale of order $R / \dot{R} \approx 5000 \mathrm{y}$. 
Early attempts to account for the pulsations in V652 Her foundered, just as they had done for the $\beta$ Cepheids (Saio 1986). Following the introduction of opacities which accounted correctly for the contribution of iron-group elements around $10^{5} \mathrm{~K}$, Saio (1993) showed that the Z-bump opacities can drive pulsations in stars like V652 Her at the observed period via the $\kappa$-mechanism. Fadeyev \& Lynas-Gray (1996) followed up these linear calculations with a non-linear analysis which found best agreement with the observed light and velocity curves for a mass of $0.72 \mathrm{M}_{\odot}$, a luminosity $1062 \mathrm{~L}_{\odot}$ and metallicity $Z=0.0156$. V652 Her must be sufficiently metal-rich for Z-bump driving to occur. Similar stars that are metal-poor do not show pulsations (e.g. HD 144941, Jeffery \& Hill 1996).

Crucial to an understanding of both the pulsations and the evolutionary status of V652 Her is a reliable measurement of its surface gravity and composition. An analysis based on a phase-averaged spectrum deduced a surface hydrogen abundance of $1 \%$ (by number), solar metallicity and CNO ratios typical of CNO-processed helium (Jeffery, Hill, \& Heber 1999). It should be recalled that V652 Her rises and falls in effective temperature by about $4000 \mathrm{~K}$ within $2.5 \mathrm{~h}$. Spectra for quantitative analysis must therefore be of short exposure and high signal-to-noise and resolution. Consequently questions persisted regarding the surface properties of V652 Her.

\section{Spectroscopic Analysis}

A sequence of 59 short exposure $(100 \mathrm{~s})$ high-quality spectra obtained in 1998 formed the basis of a new high-resolution analysis of V652 Her (Jeffery, Woolf, \& Pollacco 2001). Automatic procedures were developed to compute and fit synthetic spectra to these observations. Expansion velocity, effective temperature $T_{\text {eff }}$ and effective surface gravity $g_{\text {eff }}$ were measured as a function of pulsation phase $\phi$ directly from the optical spectra.

It is important to be able to convert the observed radial velocity curve, which represents the disk-averaged motion of the stellar surface projected into the line of sight, into the actual expansion velocity of the stellar photosphere. This projection factor is a function of the limb darkening and hence of $T_{\text {eff }}$ and composition. It turns out to be about $5 \%$ larger for stars like V652 Her than for Cepheids (Montañés Rodríguez \& Jeffery 2001).

The gravity measurements were good enough that it was possible to resolve the change in gravity due to the acceleration of the atmosphere by the pulsations $\left(\mathrm{d}^{2} R / \mathrm{d} t^{2}\right)$ and due to the change in radius of the star $\left(G M / R^{2}\right)$. A detailed abundance analysis was carried out using the sum of ten spectra near maximum radius. The quality of the data and the theoretical fit is illustrated in Fig. 1.

An interesting possibility to measure the radius directly uses only the integrated velocity curve and the changes in true gravity. If subscript 0 defines some reference phase, then the integrated velocity curve gives $\delta r(\phi)=r(\phi)-r_{0}$. From the surface gravity we have $\sqrt{g_{0} / g(\phi)}-1 \equiv\left(r(\phi)-r_{0}\right) / r_{0}$, allowing $r_{0}$ to be found by regression. However, the errors in $g(\phi)$ must be very small before this method gives a useful result.

Knowing $T_{\text {eff }}(\phi)$ and having theoretical flux distributions, a visual light curve (e.g. Kilkenny \& Lynas-Gray 1982) may be used to infer angular diameters $\theta$ from whence $r_{0}=\delta r \theta_{0} / \delta \theta$. This measure of $\theta$ is heavily dependent 


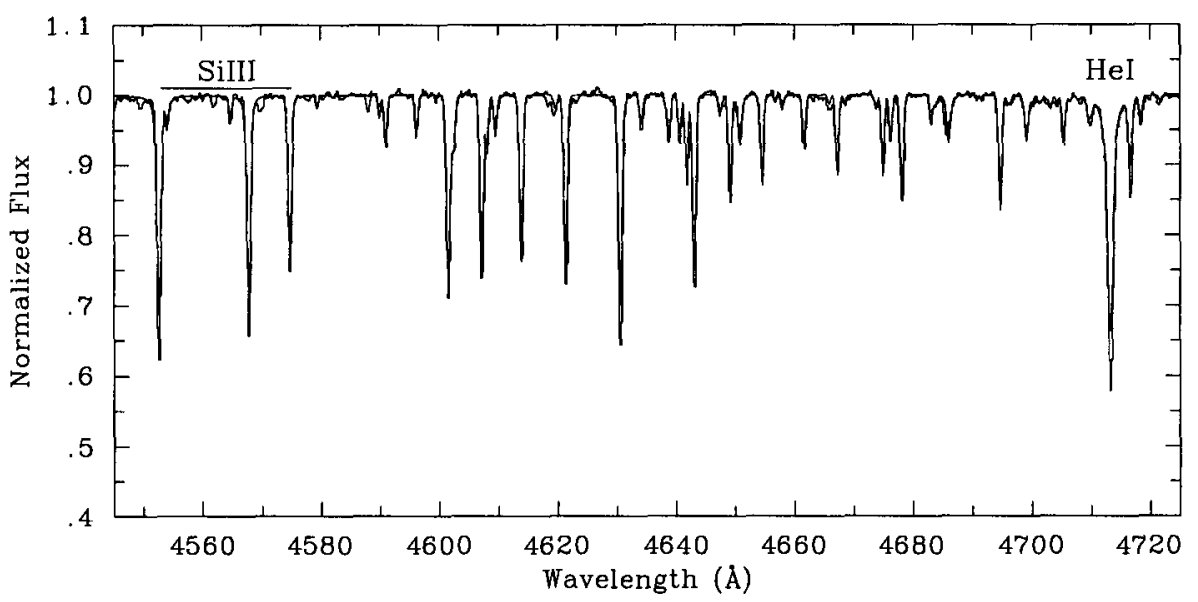

Figure 1. Part of the average normalized spectrum of V652 Her near maximum radius together with the best fit model spectrum (smooth curve) (from Jeffery et al. 2001).

on the model flux distribution and would be more reliable if based on an infrared light curve. The latter is urgently required. Another approach uses the ultraviolet and visual fluxes to measure $T_{\text {eff }}$ and $\theta$ directly (cf. Lynas-Gray et al. 1984). There remains a systematic difference of about $2000 \mathrm{~K}$ between the spectroscopic and spectrophotometric measurements of $T_{\text {eff }}$ that suggests there are still problems with the model atmospheres for early-type hydrogen-deficient giants. The ultraviolet light curve is also poorly defined near minimum radius. A consequence is that the radius measured from spectroscopy and the visual light curve is a factor of two smaller than that measured from spectroscopy and spectrophotometry. We have adopted the latter which gives an average radius for $\mathrm{V} 652 \mathrm{Her}\langle R\rangle=2.31 \pm 0.02 \mathrm{R}_{\odot}$ and leads to a mass $0.59 \pm 0.18 \mathrm{M}_{\odot}$. This is not a comfortable choice, but it provides a secure set of stellar parameters to compare directly with stellar evolution models.

\section{Pulsation models}

For radially pulsating stars, hydrodynamic models can provide an estimate of the shape and amplitude of the surface displacement and luminosity, as well as the pulsation period. These can be used to provide an additional check on the measured properties of V652 Her (cf. Fadeyev \& Lynas-Gray 1996).

In view of the revised measurements of $T_{\text {eff }}$ and $L$ (Jeffery et al. 2001), Montañés Rodríguez \& Jeffery (2002) computed new hydrodynamic models for V652 Her and the C-rich extreme helium star BX Cir, which also pulsates radially with a period of $0.10 \mathrm{~d}$. The object was to choose models that match the pulsation periods (which define the stellar radius) and then to locate the models, as a function of $M, T_{\text {eff }}$ and composition, that best reproduced the observed light and velocity curves. 

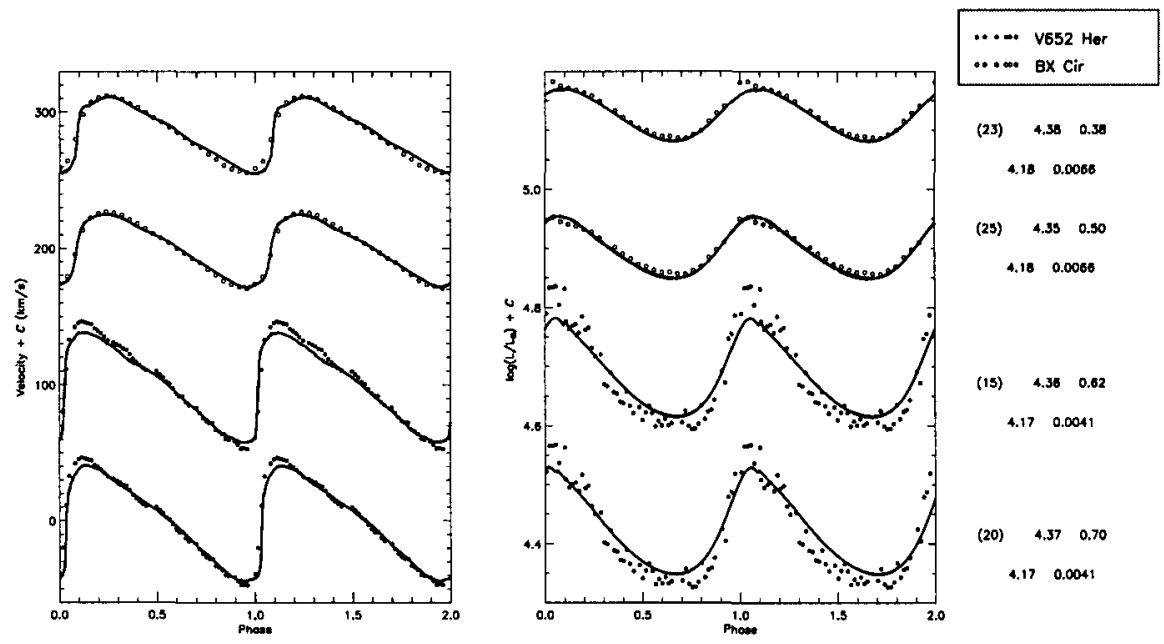

Figure 2. Pulsation models for the extreme helium stars V652 Her (bottom) and BX Cir (top) compared with the observed radial velocity and luminosity curves (from Montañés Rodríguez \& Jeffery 2002). Numbers show $\log T_{\text {eff }}, M, n_{\mathrm{Fe}} .10^{5}$ and $n_{\mathrm{Z}}$ respectively.

Good agreement was obtained for both stars (Fig. 2), although the fits to both velocity and luminosity near minimum radius are less satisfactory for V652 Her than they are for BX Cir. V652 Her shows larger amplitude pulsations so that measurements near minimum radius may be more severely affected by departures from hydrostatic and local thermodynamic equilibrium approximations. Nevertheless, these best-fit models are close to those deduced from spectroscopic and spectrophotometric analyses.

\section{Evolution Models}

V652 Her poses a unique set of problems to stellar evolution theory. It is a low-mass giant and a single star with a CNO-processed surface. It is not luminous enough to be on the evolutionary track for post-asymptotic giant branch stars, where many other hydrogen-deficient remnants are found. Jeffery (1984) suggested that it might be an unusual horizontal branch star with an envelope supported by hydrogen-burning in a very hydrogen-poor layer. His model reproduced $M, L$ and $\mathrm{d} P / \mathrm{d} t$ but did not have a plausible progenitor. Sweigart (1997) suggested similar models for extreme horizontal-branch stars based on helium-mixing on the red-giant branch, although his models were not developed with V652 Her in mind. Iben \& Tutukov (1984) argued for binary mass transfer, but V652 Her is not a binary. The luminosity and carbon abundances rule out a late helium-flash in a carbon-oxygen white dwarf.

Merged helium white dwarf models have been considered to give rise to some helium main-sequence and subdwarf B stars (e.g. Iben 1990). Saio \& 


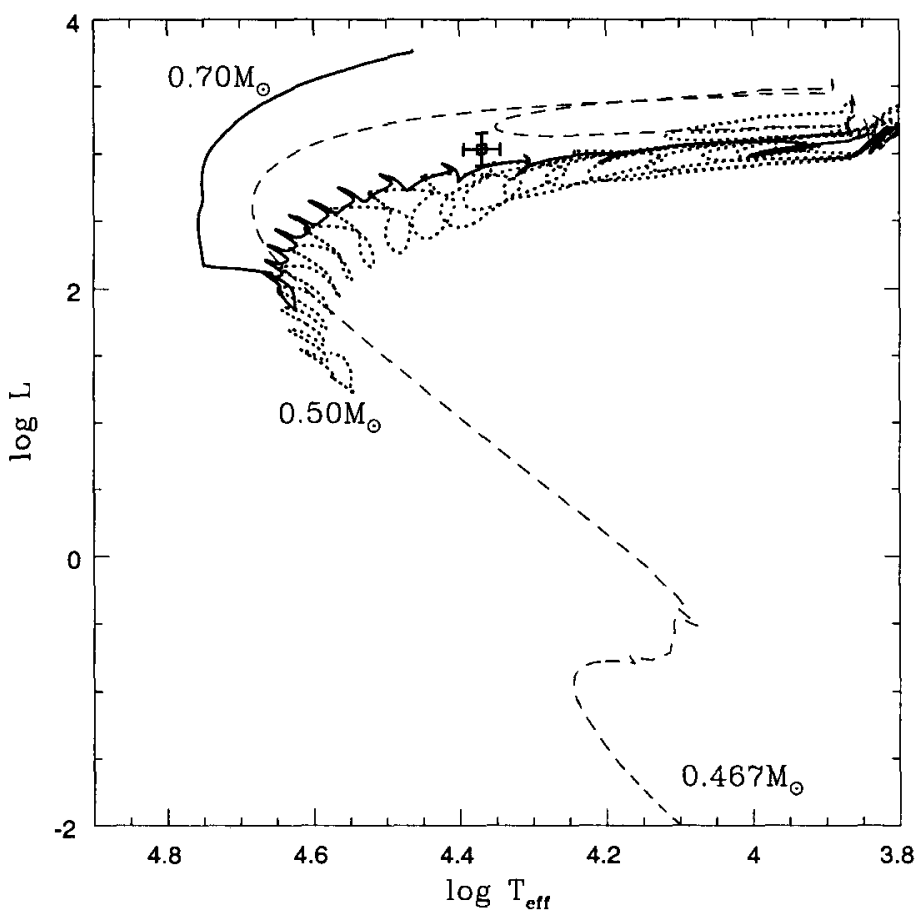

Figure 3. Evolutionary sequences for the merger of two helium white dwarfs in which the more massive white dwarf had a mass of $0.4 \mathrm{M}_{\odot}$ and the final masses were 0.5 and $0.7 \mathrm{M}_{\odot}$ (from Saio \& Jeffery 2000 ). The observed position of V652 Her is marked.

Jeffery (2000) made detailed calculations for the evolution of a star formed from the merger of two helium white dwarfs. These are achieved by accreting helium on to the surface of a $0.4 \mathrm{M}_{\odot}$ helium white dwarf. A helium-burning shell is ignited at the interface between the degenerate helium core and the non-degenerate accreted helium when the total mass is $0.467 \mathrm{M}_{\odot}$. A runaway explosion is avoided because the core is too cool. Accretion is switched off when the total mass reaches some defined value. The helium-burning shell makes the star expand to become a giant in $100-1000 \mathrm{y}$ following He ignition. It also lifts the degeneracy of the outer layers of the helium core, allowing the shell (or flame) to move inwards in mass. After the initial expansion, the flame burns inwards and decreases in power until it reaches the stellar core, whereupon the star becomes a helium main-sequence star. The surface layers respond to the inward migration of the helium-burning shell by a series of contractions and expansions. During this phase, the evolutionary tracks of stars with a final mass $0.7 \mathrm{M}_{\odot}$ pass very close to the observed location of $\mathrm{V} 652 \mathrm{Her}$ in $M, T_{\text {eff }}$ and $L$ (Fig. 3).

Pulsation periods $I$ may be found from a linear analysis of each model on an evolutionary sequence. Hence period changes $\mathrm{d} \Pi / \mathrm{d} t$ may be computed. The 
white dwarf merger models provide just the right values for $\Pi$ and $\mathrm{d} \Pi / \mathrm{d} t$ to match the observations of V652 Her (Saio \& Jeffery 2000).

Consequently, a merged binary helium white dwarf model agrees very well with the observations of V652 Her in terms of mass, luminosity, temperature, surface composition, pulsation period and contraction rate. There is no other model which achieves agreement in all of these properties.

\section{Conclusion}

V652 Her presents itself as an exotic star because of its unusual surface composition. Other properties allow it to be studied in considerable detail. The evidence that V652 Her is the product of a merger between two helium white dwarfs is strong. It lends weight to the hypothesis that white dwarf mergers really do happen and that they produce a range of other exotic stars, including some subdwarf B stars and other hydrogen-deficient remnants.

Acknowledgments. The author is indebted to coworkers Hideyuki Saio, Vincent Woolf, Don Pollacco and Pilar Montañés Rodríguez who collaborated on the work reviewed here.

\section{References}

Berger, J., \& Greenstein, J. L. 1963, PASP, 75, 336

Fadeyev, Yu. A., \& Lynas-Gray, A. E. 1996, MNRAS, 280, 427

Hill, P. W., Kilkenny, D., Schönberner, D., \& Walker, H. J. 1981, MNRAS, 197, 81

Iben, I. 1990, ApJ, 353, 215

Iben, I., \& Tutukov, A. V. 1984, ApJS, 55, 335

Jeffery, C. S. 1984, MNRAS, 210, 731

Jeffery, C. S., \& Hill, P. W. 1996, The Observatory, 116, 156

Jeffery, C. S., Hill, P. W., \& Heber, U. 1999, A\&A, 346, 491

Jeffery, C. S., Woolf, V. M., \& Pollacco, D. 2001, A\&A 376, 497

Kilkenny, D., \& Lynas-Gray, A. E. 1982, MNRAS, 198, 873

Kilkenny, D., Lynas-Gray, A. E., \& Roberts, G. 1996, MNRAS, 283, 1349

Landolt, A. U. 1975, ApJ, 196, 787

Lynas-Gray, A. E., Schönberner, D., Hill, P. W., \& Heber, U. 1984, MNRAS, 209,387

Montañès Rodríguez, P., \& Jeffery, C. S. 2001, A\&A, 375, 411

Montañès Rodríguez, P., \& Jeffery, C. S. 2002, A\&A, 384, 433

Saio, H. 1986, MNRAS, 221, 1P

Saio, H. 1993, MNRAS, 260, 465

Saio, H., \& Jeffery, C. S. 2000, MNRAS, 313, 671

Sweigart, A. V. 1997, in The Third Conference on Faint Blue Stars, ed. A. G. Davis Philip, J. W. Liebert, \& R. A. Saffer (Schenectady: L. Davis Press), 3 